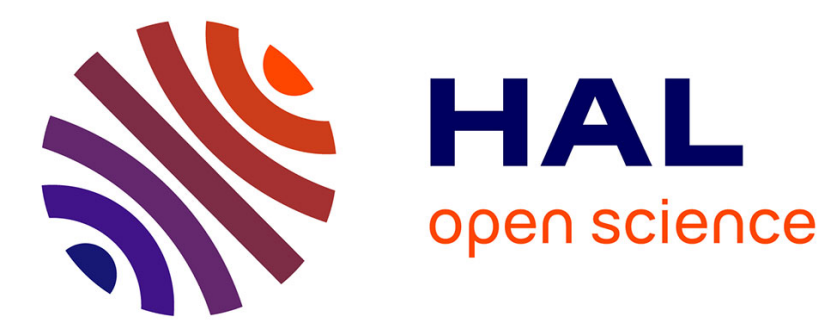

\title{
Blending constraints for composite laminates in polar parameters space
}

Enrico Panettieri, Marco Montemurro, Anita Catapano

\section{To cite this version:}

Enrico Panettieri, Marco Montemurro, Anita Catapano. Blending constraints for composite laminates in polar parameters space. Composites Part B: Engineering, 2019, 168, pp.448-457. 10.1016/j.compositesb.2019.03.040 . hal-02945353

\section{HAL Id: hal-02945353 https://hal.science/hal-02945353}

Submitted on 22 Sep 2020

HAL is a multi-disciplinary open access archive for the deposit and dissemination of scientific research documents, whether they are published or not. The documents may come from teaching and research institutions in France or abroad, or from public or private research centers.
L'archive ouverte pluridisciplinaire HAL, est destinée au dépôt et à la diffusion de documents scientifiques de niveau recherche, publiés ou non, émanant des établissements d'enseignement et de recherche français ou étrangers, des laboratoires publics ou privés. 


\title{
Blending constraints for composite laminates in polar parameters space
}

\author{
Enrico Panettieri ${ }^{\mathrm{a}}$, Marco Montemurro ${ }^{\mathrm{a}, *}$, Anita Catapano ${ }^{\mathrm{b}}$ \\ ${ }^{a}$ Arts et Métiers ParisTech, I2M CNRS UMR 5295, F-33400 Talence, France \\ ${ }^{b}$ Bordeaux INP, Université de Bordeaux, I2M CNRS UMR 5295, F-33400 Talence, France
}

\begin{abstract}
This work proposes a new formulation of blending constraints, in the framework of the multi-scale two-level (MS2L) optimisation strategy for composites. This approach aims to optimise simultaneously both geometrical and mechanical parameters of the laminate at each characteristic scale (macroscopic and mesoscopic ones). In particular, this study deals with the first level of the MS2L optimisation strategy which focuses on the laminate macroscopic scale. At this scale, the behaviour of the laminate is described in terms of the polar parameters of each constitutive stiffness matrix in the framework of the Firstorder Shear Deformation Theory (FSDT). Therefore, blending constraints are formulated as equivalent mechanical requirements to be imposed to both polar and geometric parameters of the laminate within the first-level problem. The effectiveness of the proposed approach is tested on a meaningful benchmark: the least-weight design of a composite wing-box subject to constraints of different nature. The optimised solutions provided by the MS2L design strategy are characterised by a weight saving of about $12 \%$ (when compared to the reference solution taken from the literature) by meeting the full set of feasibility, manufacturing and mechanical requirements.
\end{abstract}

Keywords: Blending constraints, ply-drop, composite materials, optimisation, finite element method, polar method

This is a pre-print of an article published in Composites Part B: Engineering.

The final authenticated version is available online at:

https://doi.org/10.1016/j.compositesb.2019.03.040

\footnotetext{
${ }^{*}$ Corresponding author.

Email address: marco.montemurro@ensam.eu; marco.montemurro@u-bordeaux.fr (Marco Montemurro )
} 


\section{Introduction}

The last three decades have witnessed a remarkable increase in the use of composite materials within aircraft structures [1]. Furthermore, the use of composite materials allows for tailoring the stiffness and the strength of the structure according to given loading conditions. In classic straight fibres composites, the tailoring can be obtained by opportunely tuning the design variables of the stack, e.g. the number of plies and the corresponding orientation, by adopting a suitable "ply drops and add" technique.

The buckling and post-buckling behaviour of tailored composites is analysed in [2-4]: the design of the structure is improved through the redistribution of zero-degree plies across the plate width. Thanks to this tailoring technique the buckling load can be increased up to $138-195 \%$, depending on the loading condition and on the laminate stack.

Following this idea, Papadopoulos and Kassapoglou [5, 6] proposed a different solution to tailor composite plates consisting of multiple concentric rectangular layups in order to maximise the buckling due to a pure shear load applied to the structure.

Kristinsdottir et al. [7] presented a work on the optimal design of composite plates subject to varying loads wherein the methodology called blending is introduced for the first time. The term blending identifies a manufacturing constraint to be considered when designing tailored composite plates. In particular, in [7] a suitable optimisation strategy implemented in the code COSTADE [8] is presented. This design strategy makes use of the ply add and drop technique during the optimisation process in order to get optimised and manufacturable laminates. This last requirement is satisfied through an opportune formulation of the previous manufacturing constraints which are called blending constraints. This concept is of paramount importance when designing large structures where the loads are usually not uniformly distributed. In this case, in order to achieve an optimised solution, the structure can be partitioned in smaller sub-structures where the loads can be considered as uniform. Due to the presence of different external loads, the optimal solution of the partitioned structure will result, in general, in different geometrical properties of each sub-structure. The problem of the optimisation of a composite structure in presence of blending constraints has been addressed in a relatively small number of works [9-15]. Constraints are typically integrated into the design process through blending rules which are imposed to the stacking sequences of adjacent laminates $[9,12,16,17]$ or that exploit relationships between the lamination parameters (LPs) at the laminate macroscopic scale (regardless of the stacking sequence) [11,13]. Among the recent works on optimisation of tailored composites, Macquart et al. [11] presented a first attempt to formulate the blending constraints in LPs space in the framework of a multi-scale optimisation strategy for composite structures.

Regardless of the adopted optimisation strategy, all the previous works share a common point: the use of pre-defined stacking sequence tables (SSTs) to retrieve a suitable laminate lay-up. More precisely, a pre-defined SST is used for the thickest laminate, then the plies are removed when moving towards the adjacent (thinner) regions in order to determine the best compromise between the set of imposed blending rules and the optimum value of the LPs determined at the laminate macroscopic scale. Of course, when passing from a thinner sub-laminate to a thicker one plies are added according to the same strategy. However, the evident drawback of such an approach is related to the choice of the initial SST which strongly shrunk the design space.

In literature, the optimisation of composite structures has been addressed by means of several approaches which can be split into two groups. The first family includes those strategies wherein the laminate is optimised by explicitly modelling the plies and the stacking sequence: examples can be found in $[9,12]$. The main drawback of this approach is that 
the designer has to deal with a strongly non-convex optimisation problem characterised by a huge number of design variables. In order to simplify the problem, several hypotheses are generally used, e.g. the use of symmetric, balanced stacks with plies orientations limited to $0, \pm 45,90$ degrees. The second group of approaches essentially includes multi-scale strategies (MSSs) [11, 18-22]. At the first stage, the global stiffness parameters of the laminate are used to describe (and optimise) the laminate behaviour at the macroscopic scale (which is modelled as an equivalent single layer plate). Then in the second step, at least one suitable stacking sequence is determined meeting the optimum values of the laminate mechanical properties resulting from the first step.

In the framework of the MSSs, the macroscopic behaviour of the laminate is defined using two main representations: the LPs and the polar parameters (PPs) [18-20, 23]. The use of these representations instead of the classical Cartesian one is linked to the anisotropy of the material. Indeed, these representations allow for describing the mechanical response of the material regardless of the considered reference frame. Moreover, LPs and PPs allow for better highlighting some physical aspects that cannot be easily caught when using the Cartesian representation. However, the representation based on LPs shows a main drawback: LPs are not tensor invariants and do not have a simple and immediate physical meaning. Conversely, PPs are true tensor invariants having an immediate physical meaning which is linked to the different (elastic) symmetries of the stiffness tensors of the laminate [24-28].

This work focuses on the optimisation of taylored composite structures by using MSSs and in particular on the multiscale two-level (MS2L) optimisation strategy initially presented in [18-20, 23]. More precisely, the present study focuses only on the first level of the MS2L optimisation method. In this background, a proper and general formulation of the blending constraints is proposed in the framework of the polar formalism and directly integrated within the first-level problem formulation. In this way, the second-level problem (the lay-up design) can be formulated as an unconstrained minimisation problem because all the requirements (geometrical, technological, mechanical, etc.) are satisfied since the first step of the MS2L optimisation strategy [18-20, 23]. The MS2L strategy relies on the use of the polar formalism extended to the case of high-order theories [26-28] for the description of the anisotropic behaviour of the composite as well as on the use of a particular genetic algorithm (GA) able to deal with optimisation problems defined over a domain of variable dimension [29]. Furthermore, in this work a general global/local modelling approach is integrated in the MS2L optimisation strategy in order to properly compute both global and local requirements to be integrated as optimisation constraints into the problem formulation. The effectiveness of the proposed formulation as well as that of the MS2L optimisation strategy is proven by means of a meaningful example taken from literature $[12,13]$ : the least-weight design of a composite wing-box structure subject to requirements of different nature.

The paper is organised as follows. In Section 2 the design problem is briefly introduced. In Section 3 the main features of the MS2L optimisation strategy are pointed out. Section 4 presents the formulation of the first-level problem in the context of the MS2L approach. In particular, the polar analysis of the First-order Shear Deformation Theory (FSDT) is briefly recalled and the analytical derivation of the blending constraints is deeply discussed. Section 5 focuses on the details of the FE model at both global and local levels, while in Section 6 the numerical results on the considered benchmark are presented and discussed. Finally, Section 7 ends the paper with some concluding remarks and perspectives. 


\section{Problem description: simplified wing-box structure}

In order to assess the blending constraints formulation based on PPs, a numerical benchmark, taken from [12, 13], has been considered in this study. The benchmark consists of a simplified wing-box model made of composite laminates subjected to an uneven distribution of forces at one end and clamped on the other one.

The geometry of the structure, the boundary conditions (BCs) and the applied loads are presented in Figure 1. The wing-box has a length of $3543 \mathrm{~mm}$, a width of $2240 \mathrm{~mm}$ and an height of $381 \mathrm{~mm}$. The wing-box is clamped on one side and four concentrated forces are applied at the middle of the opposite rib. The magnitudes of the forces are $F_{1}=90009.77$ $\mathrm{N}, F_{2}=187888.44 \mathrm{~N}, F_{3}=187888.44 \mathrm{~N}$ and $F_{4}=380176.16 \mathrm{~N}$.

In the simplified wing-box, ribs, spars and stringers are replaced by continuous equallyspaced composite plates with a fixed stacking sequence $\left[\left( \pm 45^{\circ}\right)_{11}\right]_{S}$. All laminates are made of T300/N5208 graphite-epoxy laminae whose mechanical properties are shown in Table 1 in terms of the usual cartesian value and the corresponding polar ones. The elastic properties of Table 1 are given with respect to the $x$-axes of three global laminate coordinate systems: $\Gamma^{L_{1}}=\left\{\mathrm{O} ; x_{L_{1}}, y_{L_{1}}, z_{L_{1}}\right\}, \Gamma^{L_{2}}=\left\{\mathrm{O} ; x_{L_{2}}, y_{L_{2}}, z_{L_{2}}\right\}$ and $\Gamma^{L_{3}}=\left\{\mathrm{O} ; x_{L_{3}}, y_{L_{3}}, z_{L_{3}}\right\}$. The first coordinate system is associated with both the bottom panels and top ones, the second one is used to define the stacks of both the spars and the stringers while the third one is used to correctly assign the stacks to the ribs.

\begin{tabular}{c|cc||c|cc}
\hline \multicolumn{6}{c}{ Lamina cartesian parameters } \\
\hline$E_{1}$ & 127.56 & $G P a$ & $E_{2}=E_{3}$ & 13.03 & $G P a$ \\
$G_{12}=G_{13}$ & 6.41 & $G P a$ & $\nu_{12}=\nu_{13}$ & 0.3 & - \\
$G_{23}$ & 4.5 & $G P a$ & $\nu_{23}$ & 0.45 & - \\
\hline \multicolumn{5}{c}{ Lamina polar parameters } \\
\hline \multicolumn{5}{c}{ Parameters of $[\mathrm{Q}]^{a}$} & Parameters of $[\widehat{\mathrm{Q}}]^{b}$ \\
\hline$T_{0}$ & 19.972 & $G P a$ & $T$ & 5.455 & $G P a$ \\
$T_{1}$ & 18.781 & $G P a$ & $R$ & 0.955 & $G P a$ \\
$R_{0}$ & 13.562 & $G P a$ & $\Phi$ & $\pi / 2$ & - \\
$R_{1}$ & 14.418 & $G P a$ & & \\
$\Phi_{0}=\Phi_{1}$ & 0 & - & & \\
\hline Material density, $\rho$ & 1577.76 & $\mathrm{~kg} / \mathrm{m}^{3}$ & Ply thickness, $t$ & $0.127 \mathrm{~mm}$ \\
\hline${ }^{a}$ In-plane reduced stiffness matrix of the lamina. \\
${ }^{b}$ Out-of-plane shear stiffness matrix of the lamina.
\end{tabular}

Table 1: Mechanical properties of the T300/N5208 lamina.

Different optimisation strategies of the wing-box structure were performed in [13]. Indeed, according to the definition of the sub-panels (divisions of the dorsal or ventral macro-panel) to be optimised, different optimal configurations were obtained. In the present work, only the dorsal sub-panels variables are included into the optimisation process while those of ventral panels have been set equal to those of the reference configuration. In particular, as shown in Figure 2, the dorsal panel (which constitutes the design region) has been split into three sub-panels (panels 4, 5 and 6). The reference (optimised) configuration is taken from 


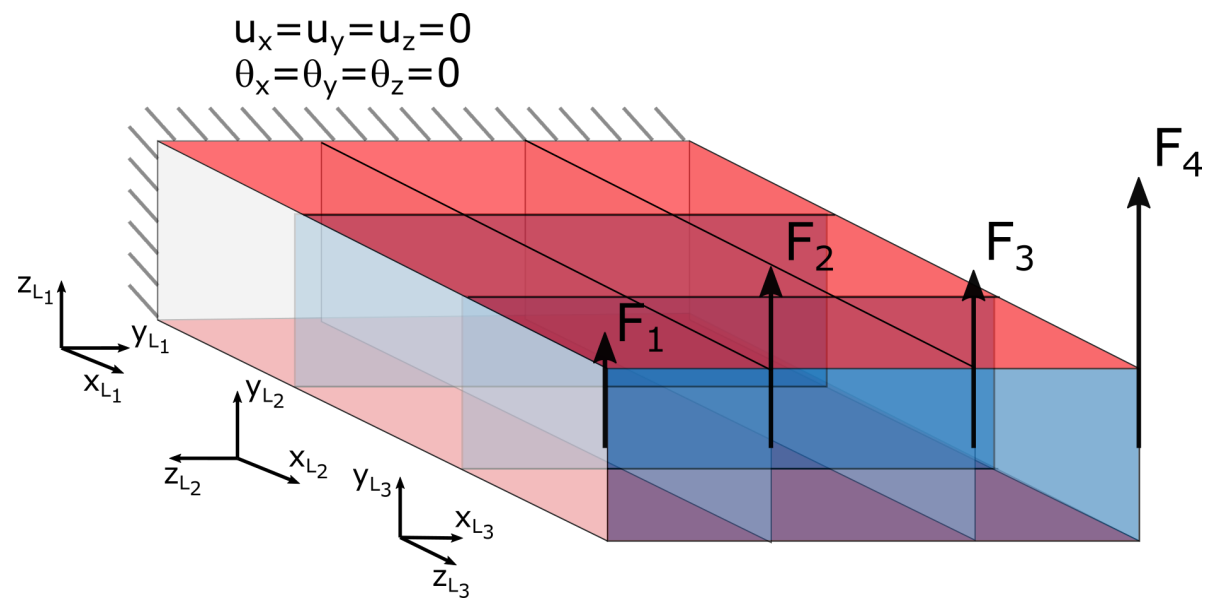

Figure 1: Wing-box geometry and boundary conditions.

[13]. For this configuration the optimum stacking sequences for panels 1-6 are reported in Table 2 .

\begin{tabular}{c|c|c}
\hline Panel ID & Stacking sequence & Number of plies \\
\hline 1 & {$\left[ \pm 45,(90)_{2},(0)_{2}\right]_{s}$} & 12 \\
2 & {$\left[ \pm 45,(90,0)_{2}, 0\right]_{s}$} & 14 \\
3 & {$\left[ \pm 45,(90,0)_{2}, 0,( \pm 45)_{2},(0)_{4},(90)_{2}, 0\right]_{s}$} & 36 \\
4 & {$\left[( \pm 45)_{2},\left((0)_{4}, 90,45,0,-45\right)_{2},\left((0)_{4}, 90,45,90,-45\right)_{2}\right.$,} & 162 \\
& $\left.(90)_{2}, 0,( \pm 45)_{3},(90, \pm 45)_{4},\left((90)_{4},(0)_{3}\right)_{3}, 90,(0)_{2}\right]_{s}$ & \\
5 & {$\left[( \pm 45)_{2},\left((0)_{4}, 90,45,0,-45\right)_{2},\left((0)_{4}, 90,45,90,-45\right)_{2}\right.$,} & 146 \\
& $\left.(90)_{2}, 0,( \pm 45)_{3},(90, \pm 45)_{4},( \pm 45)_{8}\right]_{s}$ & \\
6 & {$\left[( \pm 45)_{2},\left((0)_{4}, 90,45,0,-45\right)_{2},\left((0)_{4}, 90,45,90,-45\right)_{2}\right.$,} & \multirow{2}{*}{90} \\
& $\left.(90)_{2},(0)_{4}, 90,(0)_{2}\right]_{s}$ & \\
\hline
\end{tabular}

Table 2: Optimal stacking sequences of the sub-panels of the reference wing-box configuration taken from [13].

The reference optimal configuration presents a total weight of $314.12 \mathrm{~kg}$ and it is used as a term of comparison for the optimisation results.

\section{The multi-scale two-level optimisation strategy}

The MS2L optimisation strategy, used here to perform the least-weight design of the composite wing-box structure is articulated into two consecutive optimisation problems to be solved:

- First-level problem. The purpose of the first-level problem is the determination of the optimal values of both the PPs and the geometrical parameters of the laminates composing the structure minimising the considered cost function and satisfying the full set of design criteria. In this phase, each laminate is modelled as an equiva- 


\section{Non-design region Design region}

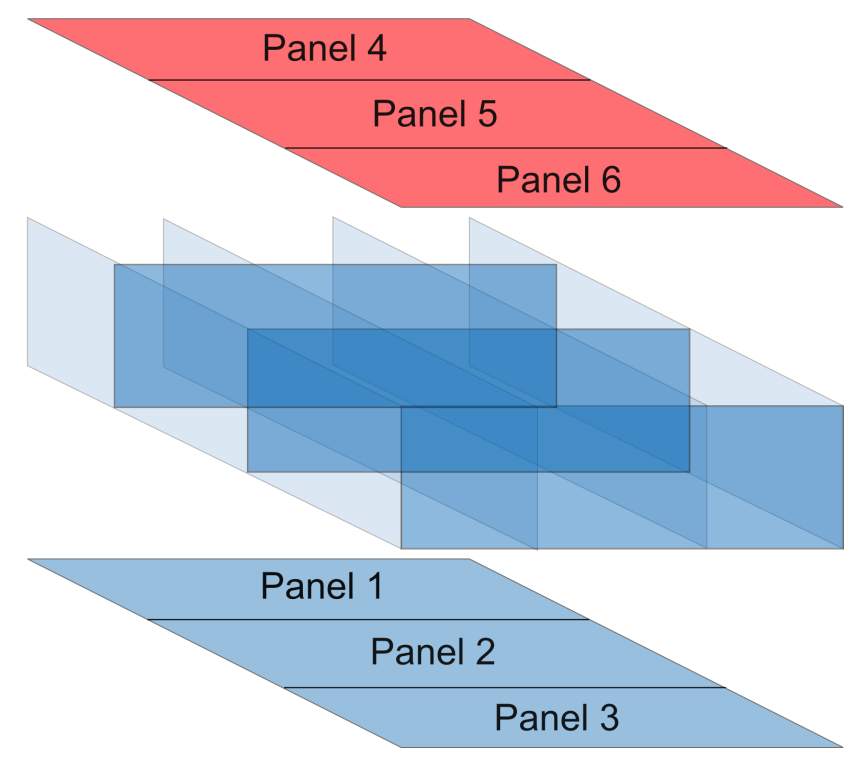

Figure 2: Design and non-design regions for the optimisation strategy.

lent homogeneous anisotropic plate whose behaviour is described through the polar formalism.

- Second-level problem. The goal of the second-level problem is to find at least one stacking sequence, for each laminate, which satisfies the optimal values of the design variables resulting from the first-level problem. The design variables of this step are the plies orientation angles.

To the best of the authors' knowledge, the blending constraints are formulated in the framework of the polar method and directly integrated into the first-level problem for the first time. Consequently, the resulting constraints are satisfied at the laminate macroscopic scale regardless of the laminate stack.

Of course, the second-level problem needs to be solved after the structural optimisation (macroscopic scale). Moreover, as discussed in [18-23, 26-28], the formulation of manufacturing requirements in terms of constraints on laminate PPs allows for considerably simplifying the second-level problem formulation. Indeed, the laminate lay-up design problem (second-level problem) can be stated in the form of an unconstrained least-squares problem in the space of layers orientation angles since manufacturing requirements are implicitly satisfied during the first optimisation phase.

\section{Mathematical formulation of the first-level problem}

The aim of the first-level problem is the mass minimisation of the three dorsal subpanels, represented in Figure 2, subject to the following design requirements:

- feasibility constraints on the laminate PPs [30];

- a constraint on the first buckling load of each sub-panel (carried out via a global-local approach); 
- a constraint on the maximum allowable strain evaluated at the centre of each subpanel;

- blending constraints on both the PPs and the number of plies for each couple of adjacent sub-panels.

Insights on these aspects are provided in the following subsections.

\subsection{Fundamentals of the polar analysis of the FSDT}

In this subsection the fundamentals of the polar analysis of the FSDT of laminates are briefly recalled. All details can be found in [26, 27]. In the framework of the FSDT the constitutive law of a multilayer plate, expressed within the global coordinate system of the laminate $\Gamma^{I}=\{\mathrm{O} ; x, y, z\}$, is:

$$
\left\{\begin{array}{c}
\{\mathrm{N}\} \\
\{\mathrm{M}\} \\
\{\mathrm{F}\}
\end{array}\right\}=\left[\begin{array}{lll}
{[\mathrm{A}]} & {[\mathrm{B}]} & {[\mathrm{O}]} \\
{[\mathrm{B}]} & {[\mathrm{D}]} & {[\mathrm{O}]} \\
{[\mathrm{O}]} & {[\mathrm{O}]} & {[\mathrm{H}]}
\end{array}\right]\left\{\begin{array}{c}
\left\{\epsilon_{0}\right\} \\
\left\{\chi_{0}\right\} \\
\left\{\gamma_{0}\right\}
\end{array}\right\}
$$

where $[\mathrm{A}],[\mathrm{B}],[\mathrm{D}]$ and $[\mathrm{H}]$ are the membrane, the membrane/bending coupling, the bending and the out-of-plane shear stiffness matrices, respectively. $\{N\},\{M\}$ and $\{F\}$ represent the vectors of membrane forces, bending moments and out-of-plane shear forces per unit length, respectively. Moreover, $\left\{\epsilon_{0}\right\},\left\{\chi_{0}\right\}$ and $\left\{\gamma_{0}\right\}$ are the vectors of in-plane strains, curvatures and out-of-plane shear strains of the laminate mid-plane, respectively.

The matrices $[\mathrm{A}],[\mathrm{B}],[\mathrm{D}]$ and $[\mathrm{H}]$ depend on the in-plane and out-of-plane reduced stiffness matrices of the $k$-th ply, i.e. $\left[\mathrm{Q}\left(\delta_{k}\right)\right]$ and $\left[\widehat{\mathrm{Q}}\left(\delta_{k}\right)\right]$, respectively, on the orientation angle $\delta_{k}$ and on thickness of the $k$-th lamina as well as on the overall number of plies $N$. For a generic laminate of overall thickness $h$ constituted of $N$ identical plies (i.e. same material and thickness), the following relationships hold:

$$
\begin{array}{ll}
{[\mathrm{A}]=\frac{h}{N} \sum_{k=1}^{N}\left[\mathrm{Q}\left(\delta_{k}\right)\right],} & {[\mathrm{B}]=\frac{1}{2}\left(\frac{h}{N}\right)^{2} \sum_{k=1}^{N} b_{k}\left[\mathrm{Q}\left(\delta_{k}\right)\right],} \\
{[\mathrm{D}]=\frac{1}{12}\left(\frac{h}{N}\right)^{3} \sum_{k=1}^{N} d_{k}\left[\mathrm{Q}\left(\delta_{k}\right)\right],} & {[\mathrm{H}]=\frac{h}{N} \sum_{k=1}^{N}\left[\widehat{\mathrm{Q}}\left(\delta_{k}\right)\right],}
\end{array}
$$

where

$$
\begin{aligned}
& b_{k}=2 k-N-1, \quad \sum_{k=1}^{N} b_{k}=0, \\
& d_{k}=12 k(k-N-1)+4+3 N(N+2), \quad \sum_{k=1}^{N} d_{k}=N^{3},
\end{aligned}
$$

where the index $k$ defines the position of each ply within the laminate starting from the bottom of the stack.

For design purposes, it is very useful to normalise the units of the laminate stiffness matrices to those of the ply reduced stiffness matrices:

$$
\left[\mathrm{A}^{*}\right]=\frac{1}{h}[\mathrm{~A}], \quad\left[\mathrm{B}^{*}\right]=\frac{2}{h^{2}}[\mathrm{~B}], \quad\left[\mathrm{D}^{*}\right]=\frac{12}{h^{3}}[\mathrm{D}], \quad\left[\mathrm{H}^{*}\right]=\frac{1}{h}[\mathrm{H}] .
$$


Through the polar formalism, the components of matrices $\left[\mathrm{Q}\left(\delta_{k}\right)\right]$ and $\left[\widehat{\mathrm{Q}}\left(\delta_{k}\right)\right]$ of Eq. (2) can be written, with respect to the lamina coordinate system $\Gamma=\left\{\mathrm{O} ; x_{1}, x_{2}, x_{3}\right\}$, as functions of the polar parameters of the lamina [26, 27]. In particular the components of [Q $\left.\left(\delta_{k}\right)\right]$ matrix are defined through six polar parameters, of which five are tensor invariants: the isotropic moduli $T_{0}$ and $T_{1}$, the anisotropic moduli $R_{0}$ and $R_{1}$ and the difference between the polar angles $\Phi_{0}$ and $\Phi_{1}$. Similarly, the components of $\left[\widehat{\mathrm{Q}}\left(\delta_{k}\right)\right]$ matrix depend on three polar parameters: the isotropic modulus $T$, the deviatoric one $R$, and the polar angle $\Phi$ (among them only $T$ and $R$ are polar invariants).

As for the lamina, at the laminate level, the matrices $\left[\mathrm{A}^{*}\right],\left[\mathrm{B}^{*}\right],\left[\mathrm{D}^{*}\right]$ and $\left[\mathrm{H}^{*}\right]$ of Eq. (1) can be reformulated in terms of laminate polar parameters. The general mathematical formulation can be found in [26, 27]. Simplifications of the general equations can be done to address solutions that are typically used in real-life engineering applications. In particular, in this work, the hypotheses of uncoupled and fully orthotropic laminate (both membrane and bending matrices), regardless of the considered stacking sequence, are adopted. These hypotheses can be taken into account by means of the following relationships:

$$
\left[\mathrm{B}^{*}\right]=[\mathrm{O}], \Phi_{0}^{M^{*}}-\Phi_{1}^{M^{*}}=K^{M^{*}} \frac{\pi}{4}, M^{*}=A^{*}, D^{*} .
$$

Under these hypotheses, matrices $\left[\mathrm{A}^{*}\right],\left[\mathrm{D}^{*}\right]$ and $\left[\mathrm{H}^{*}\right]$ can be expressed, in terms of their polar parameters, as follows:

$$
\begin{array}{lc}
T_{0}^{A^{*}}=T_{0}, & T_{1}^{A^{*}}=T_{1}, \\
R_{0 K}^{A^{*}} e^{\mathrm{i} 4 \Phi_{1}^{A^{*}}}=\frac{1}{N} R_{0} \sum_{k=1}^{N} e^{\mathrm{i} 4 \delta_{k}}, & R_{1}^{A^{*}} e^{\mathrm{i} 2 \Phi_{1}^{A^{*}}}=\frac{1}{N} R_{1} \sum_{k=1}^{N} e^{\mathrm{i} 2 \delta_{k}}, \\
T_{0}^{D^{*}}=T_{0}, & T_{1}^{D^{*}}=T_{1}, \\
R_{0 K}^{D^{*}} e^{\mathrm{i} 4 \Phi_{1}^{D^{*}}}=\frac{1}{N^{3}} R_{0} \sum_{k=1}^{N} d_{k} e^{\mathrm{i} 4 \delta_{k}}, & R_{1}^{D^{*}} e^{\mathrm{i} 2 \Phi_{1}^{D^{*}}}=\frac{1}{N^{3}} R_{1} \sum_{k=1}^{N} d_{k} e^{\mathrm{i} 2 \delta_{k}}, \\
T^{H^{*}}=T, R^{H^{*}} e^{\mathrm{i} 2 \Phi^{H^{*}}}=\frac{1}{N} R e^{\mathrm{i} 2 \Phi} \sum_{k=1}^{N} e^{-\mathrm{i} 2 \delta_{k}} .
\end{array}
$$

In Eqs. (6) and (7), $T_{0}^{A^{*}}, T_{0}^{D^{*}}, T_{1}^{A^{*}}$ and $T_{1}^{D^{*}}$ are the isotropic moduli, $R_{0 K}^{A^{*}}, R_{0 K}^{D^{*}}, R_{1}^{A^{*}}$ and $R_{1}^{D^{*}}$ are the anisotropic ones and i is the imaginary unit, while $\Phi_{1}^{A^{*}}$ and $\Phi_{1}^{D^{*}}$ represent the direction of the main orthotropy axis of each stiffness matrix. The terms $R_{0 K}^{A^{*}}$ and $R_{0 K}^{D^{*}}$ are defined as follows:

$$
R_{0 K}^{A^{*}}=(-1)^{K^{A^{*}}} R_{0}^{A^{*}}, R_{0 K}^{D^{*}}=(-1)^{K^{D^{*}}} R_{0}^{D^{*}},
$$

with $K^{A^{*}}$ and $K^{D^{*}}$ equal to either 0 or 1 depending on the shape of the orthotropy, see $[26,27]$.

In Eq. (8), $T^{H^{*}}$ and $R^{H^{*}}$ represent the isotropic and the deviatoric moduli, respectively, and $\Phi^{H^{*}}$ the polar angle. As shown in $[26,27]$, the anisotropic part of the out-of-plane shear matrix $\left[\mathrm{H}^{*}\right]$ can be written in terms of that of $\left[\mathrm{A}^{*}\right]$ as follows:

$$
R^{H^{*}} e^{\mathrm{i} 2 \Phi^{H^{*}}}=\frac{R}{R_{1}} R_{1}^{A^{*}} e^{\mathrm{i} 2\left(\Phi+\Phi_{1}-\Phi_{1}^{A^{*}}\right)} .
$$


This is one of the main advantages of the polar formalism when applied to the FSDT. When passing from the classical laminate theory (CLT) to the FSDT the number of polar parameters to be optimised during the first-level problem remains unchanged. Therefore solely the polar parameters of $\left[\mathrm{A}^{*}\right]$ and $\left[\mathrm{D}^{*}\right]$ matrices are needed to fully describe the behaviour of the laminate at the macroscopic scale.

Eqs. (6) and (7) can be rewritten in terms of LPs which constitute an alternative representation to describe the behaviour of multilayer plates [31]:

$$
\begin{array}{rlrl}
V_{1}^{A^{*}} & =\frac{1}{N} \sum_{k=1}^{N} \cos \left(2 \delta_{k}\right), & V_{1}^{D^{*}}=\frac{1}{N^{3}} \sum_{k=1}^{N} d_{k} \cos \left(2 \delta_{k}\right), \\
V_{2}^{A^{*}} & =\frac{1}{N} \sum_{k=1}^{N} \sin \left(2 \delta_{k}\right), \quad V_{2}^{D^{*}}=\frac{1}{N^{3}} \sum_{k=1}^{N} d_{k} \sin \left(2 \delta_{k}\right), \\
V_{3}^{A^{*}} & =\frac{1}{N} \sum_{k=1}^{N} \cos \left(4 \delta_{k}\right), \quad V_{3}^{D^{*}}=\frac{1}{N^{3}} \sum_{k=1}^{N} d_{k} \cos \left(4 \delta_{k}\right), \\
V_{4}^{A^{*}} & =\frac{1}{N} \sum_{k=1}^{N} \sin \left(4 \delta_{k}\right), & V_{4}^{D^{*}} & =\frac{1}{N^{3}} \sum_{k=1}^{N} d_{k} \sin \left(4 \delta_{k}\right) .
\end{array}
$$

By using the LPs in Eq. (11) and the expression of the laminate PPs of Eqs. (6) and (7), the following relationships between LPs and PPs can be easily inferred:

$$
\begin{array}{ll}
R_{0 K}^{A^{*}} e^{\mathrm{i} 4 \Phi_{1}^{A^{*}}}=R_{0}\left(V_{3}^{A^{*}}+\mathrm{i} V_{4}^{A^{*}}\right), & R_{1}^{A^{*}} e^{\mathrm{i} 2 \Phi_{1}^{A^{*}}}=R_{1}\left(V_{1}^{A^{*}}+\mathrm{i} V_{2}^{A^{*}}\right), \\
R_{0 K}^{D^{*}} e^{\mathrm{i} 4 \Phi_{1}^{D^{*}}}=R_{0}\left(V_{3}^{D^{*}}+\mathrm{i} V_{4}^{D^{*}}\right), & R_{1}^{D^{*}} e^{\mathrm{i} 2 \Phi_{1}^{D^{*}}}=R_{1}\left(V_{1}^{D^{*}}+\mathrm{i} V_{2}^{D^{*}}\right) .
\end{array}
$$

\subsection{Blending constraints formulation}

To derive the analytical formulae defining the constraints to be imposed on the relevant laminate PPs in a general blending condition, the scheme of Figure 3 is adopted. If blending is seen as a plies drop process, two laminates are blended when a given number of plies is suppressed from a laminate in order to obtain a thinner one. For the sake of simplicity the first laminate (the one from which plies are removed) will be named thick laminate and the second one thin laminate.

Let $k_{1}$ and $k_{2}$ be the indexes identifying the stacking sequences of thick and thin laminates, respectively. Let $m$ be the number of plies removed from the thick laminate in order to obtain the thin one. Thus, the total number of plies of the thick laminate is $N$, whilst that of the thin one is $N-m$.

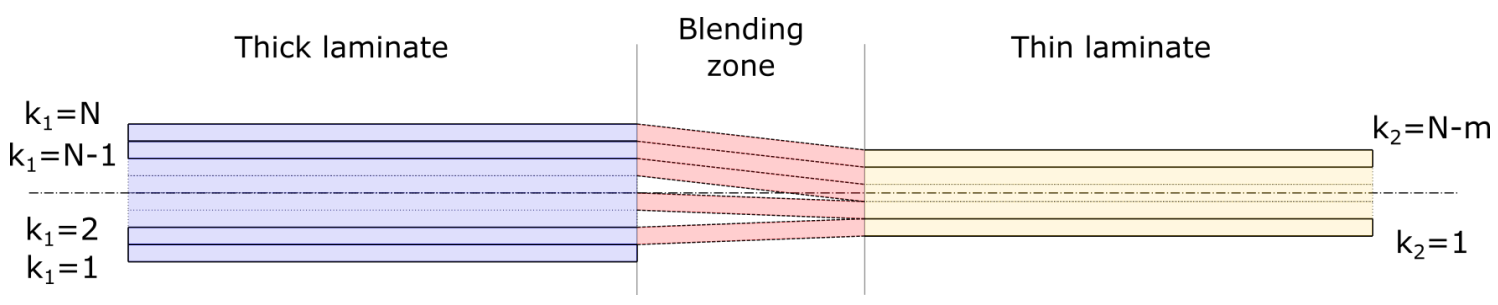

Figure 3: Laminates obtained through a generic blending strategy.

The starting point for the derivation of blending constraints is Eq. (12). In particular, the 
real and imaginary parts for the PPs of matrices $\left[\mathrm{A}^{*}\right]$ and $\left[\mathrm{D}^{*}\right]$ can be split as follows:

$$
\begin{array}{ll}
R_{0 K}^{A^{*}} \cos \left(4 \Phi_{1}^{A^{*}}\right)=R_{0} V_{3}^{A^{*}}, & R_{1}^{A^{*}} \cos \left(2 \Phi_{1}^{A^{*}}\right)=R_{1} V_{1}^{A^{*}}, \\
R_{0 K}^{A^{*}} \sin \left(4 \Phi_{1}^{A^{*}}\right)=R_{0} V_{4}^{A^{*}}, & R_{1}^{A^{*}} \sin \left(2 \Phi_{1}^{A^{*}}\right)=R_{1} V_{2}^{A^{*}}, \\
R_{0 K}^{D^{*}} \cos \left(4 \Phi_{1}^{D^{*}}\right)=R_{0} V_{3}^{D^{*}}, & R_{1}^{D^{*}} \cos \left(2 \Phi_{1}^{D^{*}}\right)=R_{1} V_{1}^{D^{*}}, \\
R_{0 K}^{D^{*}} \sin \left(4 \Phi_{1}^{D^{*}}\right)=R_{0} V_{4}^{D^{*}}, & R_{1}^{D^{*}} \sin \left(2 \Phi_{1}^{D^{*}}\right)=R_{1} V_{2}^{D^{*}} .
\end{array}
$$

In the following, blending constraints are derived independently for membrane and bending stiffness matrices.

\subsubsection{Blending constraints for the membrane stiffness matrix}

Moving from the thick laminate to the thin one, the only terms of Eq. (13) that do not change are the anisotropic moduli of the lamina $\left(R_{0}\right.$ and $\left.R_{1}\right)$. Therefore the maximum variation of the left-hand members of the Eq. (13) is related to the maximum variation of the corresponding LPs.

Thus, the maximum variation of the membrane PPs can be written as follows:

$$
\begin{aligned}
& \max \left[\Delta\left(R_{0 K}^{A^{*}} \cos \left(4 \Phi_{1}^{A^{*}}\right)\right)\right]=R_{0} \max \left(\Delta V_{3}^{A^{*}}\right), \\
& \max \left[\Delta\left(R_{0 K}^{A^{*}} \sin \left(4 \Phi_{1}^{A^{*}}\right)\right)\right]=R_{0} \max \left(\Delta V_{4}^{A^{*}}\right), \\
& \max \left[\Delta\left(R_{1}^{A^{*}} \cos \left(2 \Phi_{1}^{A^{*}}\right)\right)\right]=R_{1} \max \left(\Delta V_{1}^{A^{*}}\right), \\
& \max \left[\Delta\left(R_{1}^{A^{*}} \sin \left(2 \Phi_{1}^{A^{*}}\right)\right)\right]=R_{1} \max \left(\Delta V_{2}^{A^{*}}\right),
\end{aligned}
$$

where the operator $\Delta$ refers to a difference computed between the thick laminate ( $N$ plies) and the thin one ( $N-m$ plies).

As reported in the literature [11], it can be easily verified that the maximum variation of the LPs of the membrane stiffness matrix of Eq. (15) is equal to $2 \frac{m}{N}$. Therefore, Eq. (15) can be rewritten as follows:

$$
\begin{aligned}
g_{1 j s} & =\left|\max \left[\Delta_{j s}\left(R_{0 K}^{A^{*}} \cos \left(4 \Phi_{1}^{A^{*}}\right)\right)\right]\right|-2 R_{0} \frac{\left|N_{j}-N_{s}\right|}{\max \left(N_{j}, N_{s}\right)} \leq 0, \\
g_{2 j s} & =\left|\max \left[\Delta_{j s}\left(R_{0 K}^{A^{*}} \sin \left(4 \Phi_{1}^{A^{*}}\right)\right)\right]\right|-2 R_{0} \frac{\left|N_{j}-N_{s}\right|}{\max \left(N_{j}, N_{s}\right)} \leq 0, \\
g_{3 j s} & =\left|\max \left[\Delta_{j s}\left(R_{1}^{A^{*}} \cos \left(2 \Phi_{1}^{A^{*}}\right)\right)\right]\right|-2 R_{1} \frac{\left|N_{j}-N_{s}\right|}{\max \left(N_{j}, N_{s}\right)} \leq 0, \\
g_{4 j s} & =\left|\max \left[\Delta_{j s}\left(R_{1}^{A^{*}} \sin \left(2 \Phi_{1}^{A^{*}}\right)\right)\right]\right|-2 R_{1} \frac{\left|N_{j}-N_{s}\right|}{\max \left(N_{j}, N_{s}\right)} \leq 0,
\end{aligned}
$$

with $j=1, \cdots, N_{p}$ and $s \in \mathrm{LS}_{j}$.

In the previous equation, $N_{p}$ is the total number of sub-panels composing the design region of the structure. $\mathrm{LS}_{j}$ is the influence zone of the $j$-th sub-panel which collects all the indexes of those sub-panels adjacent to the panel $j . N_{j}$ and $N_{s}$ are the number of plies of two adjacent laminates, whilst $\Delta_{j s}(\cdots)$ represents the difference between the PPs for the generic couple $j s$ of panels. The inequalities of Eq. (16) represent the blending constraints 
to be imposed on the PPs of $\left[\mathrm{A}^{*}\right]$, when the membrane stiffness matrix has an orthotropic behaviour.

\subsubsection{Blending constraints for the bending stiffness matrix}

The derivation of the blending constraints for the bending stiffness matrix PPs is more difficult than in the case of the membrane stiffness matrix. Similarly to the previous case, the maximum variation of the left-hand members of Eq. (14) is related to the maximum variation of the corresponding LPs:

$$
\begin{aligned}
& \max \left[\Delta\left(R_{0 K}^{D^{*}} \cos \left(4 \Phi_{1}^{D^{*}}\right)\right)\right]=R_{0} \max \left(\Delta V_{3}^{D^{*}}\right), \\
& \max \left[\Delta\left(R_{0 K}^{D^{*}} \sin \left(4 \Phi_{1}^{D^{*}}\right)\right)\right]=R_{0} \max \left(\Delta V_{4}^{D^{*}}\right), \\
& \max \left[\Delta\left(R_{1}^{D^{*}} \cos \left(2 \Phi_{1}^{D^{*}}\right)\right)\right]=R_{1} \max \left(\Delta V_{1}^{D^{*}}\right), \\
& \max \left[\Delta\left(R_{1}^{D^{*}} \sin \left(2 \Phi_{1}^{D^{*}}\right)\right)\right]=R_{1} \max \left(\Delta V_{2}^{D^{*}}\right) .
\end{aligned}
$$

The analytical formulae reported in [11] to express the maximum variation of the LPs of matrix $\left[\mathrm{D}^{*}\right]$ cannot be used here because they do not correctly integrate the influence of the ply position $k$ when passing from the thick laminate to the thin one. Indeed, the LPs related to the bending behaviour of the laminate include a term, $d_{k}$, which depends on the position of the $k^{t h}$ lamina within the laminate (cfr. Eq. (2)). Moreover, the laminate reference frame changes when passing from the thick stack to the thin one. Accordingly, indices $k_{1}$ and $k_{2}$ are not the same and the determination of the relationship between terms $d_{k_{1}}$ and $d_{k_{2}}$ is not an easy task.

Only the algebraic passages related to the term $\Delta V_{1}^{D^{*}}$ are reported here below for the sake of synthesis. The same procedure can be repeated for the rest of LPs related to matrix $\left[\mathrm{D}^{*}\right]$. Of course, once the maximum variation of each LP is determined, the derivation of the maximum variation of the bending PPs is immediate, according to Eq. (17).

According to the adopted notation, the variation of the term $V_{1}^{D^{*}}$ when passing from the thick laminate to the thin one is

$$
\Delta V_{1}^{D^{*}}=\frac{1}{N^{3}} \sum_{k_{1}=1}^{N} d_{k_{1}} \cos \left(2 \delta_{k_{1}}\right)-\frac{1}{(N-m)^{3}} \sum_{k_{2}=1}^{N-m} d_{k_{2}} \cos \left(2 \delta_{k_{2}}\right),
$$

where the $m$ plies can be removed in arbitrary positions, as shown in Figure 3. In order to obtain the desired result, two additional indexes must be defined:

- $k_{m}$ is the index of the ply position within the set of the removed plies, i.e. $i_{1}, i_{2}, \ldots, i_{m}$,

- $k_{1}^{*}$ is the index of the ply position within the set of non-suppressed plies, i.e. $j_{1}$, $j_{2}, \ldots, j_{N-m}$.

By means of these additional parameters the term $V_{1}^{D^{*}}$ relative to the thick laminate can be decomposed so that Eq. (18) becomes:

$$
\begin{aligned}
\Delta V_{1 D^{*}} & =\frac{1}{N^{3}} \sum_{k_{m}=i_{1}}^{i_{m}} d_{k_{m}} \cos \left(2 \delta_{k_{m}}\right)+\frac{1}{N^{3}} \sum_{k_{1}^{*}=j_{1}}^{j_{N-m}} d_{k_{1}^{*}} \cos \left(2 \delta_{k_{1}^{*}}\right)+ \\
& -\frac{1}{(N-m)^{3}} \sum_{k_{2}=1}^{N-m} d_{k_{2}} \cos \left(2 \delta_{k_{2}}\right),
\end{aligned}
$$


where the first term of the right-hand member represents a summation performed over the set of suppressed plies and the second one is relative to the set of non-suppressed plies.

The maximum variation of $V_{1 D^{*}}$ is achieved when both the non-suppressed and the removed plies share the same orientation angle, i.e. $\delta_{k_{1}^{*}}=\delta_{k_{2}}=\delta$ and $\delta_{k_{m}}=\delta_{s}$. Eq. (19) becomes:

$$
\begin{aligned}
\max \left(\Delta V_{1 D^{*}}\right) & =\frac{\cos \left(2 \delta_{s}\right)}{N^{3}} \sum_{k_{m}=i_{1}}^{i_{m}} d_{k_{m}}+\cos (2 \delta)\left(\frac{1}{N^{3}} \sum_{k_{1}^{*}=j_{1}}^{j_{N-m}} d_{k_{1}^{*}}\right. \\
& \left.-\frac{1}{(N-m)^{3}} \sum_{k_{2}=1}^{N-m} d_{k_{2}}\right)=\frac{\cos \left(2 \delta_{s}\right)}{N^{3}} \sum_{k_{m}=i_{1}}^{i_{m}} d_{k_{m}}+ \\
& +\cos (2 \delta)\left(\frac{1}{N^{3}} \sum_{k_{1}^{*}=j_{1}}^{j_{N-m}} d_{k_{1}^{*}}-1\right) .
\end{aligned}
$$

Since the term $d_{k}$ is always non-negative, the maximum absolute value of $\Delta V_{1 D^{*}}$ is obtained for $\delta_{s}=0$ and $\delta=\frac{\pi}{2}$ (or vice versa). Accordingly, Eq. (20) further simplifies:

$$
\begin{aligned}
\left|\max \left(\Delta V_{1 D^{*}}\right)\right| & =\frac{1}{N^{3}} \sum_{k_{m}=i_{1}}^{i_{m}} d_{k_{m}}+1-\frac{1}{N^{3}} \sum_{k_{1}^{*}=j_{1}}^{j_{N-m}} d_{k_{1}^{*}}= \\
& =\frac{1}{N^{3}} \sum_{k_{m}=i_{1}}^{i_{m}} d_{k_{m}}+1-\left(\frac{1}{N^{3}} \sum_{k_{1}=1}^{N} d_{k_{1}}-\frac{1}{N^{3}} \sum_{k_{m}=i_{1}}^{i_{m}} d_{k_{m}}\right)= \\
& =\frac{2}{N^{3}} \sum_{k_{m}=i_{1}}^{i_{m}} d_{k_{m}} .
\end{aligned}
$$

This result shows that the maximum absolute of $\Delta V_{1 D^{*}}$ depends on the position of the suppressed plies within the thick stack. The summation $\sum_{k_{m}=i_{1}}^{i_{m}} d_{k_{m}}$ can be further simplified as follows:

$$
\sum_{k_{m}=i_{1}}^{i_{m}} d_{k_{m}}=m\left(4+6 N+3 N^{2}\right)+12 \sum_{k_{m}=i_{1}}^{i_{m}} f\left(k_{m}\right),
$$

where $f\left(k_{m}\right)=k_{m}^{2}-N k_{m}-k_{m}$. The value assumed by the $f\left(k_{m}\right)$ function depends on the position of the removed layers. It is clear that the maximum value of $\sum_{k_{m}=i_{1}}^{i_{m}} f\left(k_{m}\right)$ is obtained when the suppressed plies are at the extreme positions within the stack. The most critical case occurs when plies are removed symmetrically with respect to the mid-plane of the thick laminate. Depending on the value of $m$ two cases can occur.

\section{- Even value of $m$}

In this case the suppressed plies are symmetrically grouped at the top and at the bottom of the laminate. Introducing the quantity $M_{s}=m / 2$, Eq. (22) reads:

$$
\sum_{k_{m}=i_{1}}^{i_{m}} f\left(k_{m}\right)=\frac{2}{3} M_{s}^{3}-N M_{s}^{2}-M_{s}\left(N+\frac{2}{3}\right)
$$

\section{- Odd value of $m$}


In this case, $(m-1) / 2$ suppressed plies will be grouped at one side of the laminate and the remaining $(m-1) / 2+1$ suppressed plies will be grouped on the other side. In this case the parameter $M_{s}$ is defined as $M_{s}=(m-1) / 2$, then Eq. (22) reads:

$$
\sum_{k_{m}=i_{1}}^{i_{m}} f\left(k_{m}\right)=\frac{2}{3} M_{s}^{3}-N M_{s}^{2}-M_{s}\left(N+\frac{2}{3}\right)+\left(M_{s}+1\right)\left(M_{s}-N\right) .
$$

Finally the maximum absolute variation of $\left|\max \left(\Delta V_{1 D^{*}}\right)\right|$ can be expressed as:

$$
\left|\max \left(\Delta V_{1 D^{*}}\right)\right|=\frac{2}{N^{3}}\left(m\left(4+6 N+3 N^{2}\right)+12 \sum_{k_{m}=i_{1}}^{i_{m}} f\left(k_{m}\right)\right)=\frac{2}{N^{3}} S_{k_{m}},
$$

with

$$
S_{k_{m}}=S_{k_{m}}(N, m)=m\left(4+6 N+3 N^{2}\right)+12 \sum_{k_{m}=i_{1}}^{i_{m}} f\left(k_{m}\right) .
$$

The term $\sum_{k_{m}=i_{1}}^{i_{m}} f\left(k_{m}\right)$ must be evaluated according to the value of the number of the suppressed plies, $m$, as reported in Eqs. (23) and (24).

The same results can be obtained for the other LPs of the [D*] matrix of Eq. (17) with the only difference that the maximum absolute value of the terms $\Delta V_{2}^{D^{*}}, \Delta V_{3}^{D^{*}}$ and $\Delta V_{4}^{D^{*}}$ occur for different combinations of the orientations of removed and non-suppressed plies:

- $\delta_{s}=\frac{\pi}{4}$ and $\delta=-\frac{\pi}{4}$ for $\Delta V_{2}^{D^{*}}$,

- $\delta_{s}=0$ and $\delta=\frac{\pi}{4}$ for $\Delta V_{3}^{D^{*}}$,

- $\delta_{s}=\frac{\pi}{8}$ and $\delta=-\frac{\pi}{8}$ for $\Delta V_{4}^{D^{*}}$.

Finally, the blending constraints on the PPs of matrix $\left[\mathrm{D}^{*}\right]$ can be stated as follows:

$$
\begin{aligned}
& g_{5 j s}=\left|\max \left[\Delta_{j s}\left(R_{0 K}^{D^{*}} \cos \left(4 \Phi_{1}^{D^{*}}\right)\right)\right]\right|-2 \frac{R_{0}}{\max \left(N_{j}^{3}, N_{s}^{3}\right)} S_{k_{m}}\left(\max \left(N_{j}, N_{s}\right),\left|N_{j}-N_{s}\right|\right) \leq 0, \\
& g_{6 j s}=\left|\max \left[\Delta_{j s}\left(R_{0 K}^{D^{*}} \sin \left(4 \Phi_{1}^{D^{*}}\right)\right)\right]\right|-2 \frac{R_{0}}{\max \left(N_{j}^{3}, N_{s}^{3}\right)} S_{k_{m}}\left(\max \left(N_{j}, N_{s}\right),\left|N_{j}-N_{s}\right|\right) \leq 0, \\
& g_{7 j s}=\left|\max \left[\Delta_{j s}\left(R_{1}^{D^{*}} \cos \left(2 \Phi_{1}^{D^{*}}\right)\right)\right]\right|-2 \frac{R_{1}}{\max \left(N_{j}^{3}, N_{s}^{3}\right)} S_{k_{m}}\left(\max \left(N_{j}, N_{s}\right),\left|N_{j}-N_{s}\right|\right) \leq 0, \\
& g_{8 j s}=\left|\max \left[\Delta_{j s}\left(R_{1}^{D^{*}} \sin \left(2 \Phi_{1}^{D^{*}}\right)\right)\right]\right|-2 \frac{R_{1}}{\max \left(N_{j}^{3}, N_{s}^{3}\right)} S_{k_{m}}\left(\max \left(N_{j}, N_{s}\right),\left|N_{j}-N_{s}\right|\right) \leq 0, \\
& \text { with } j=1, \cdots, N_{p} \text { and } s \in \mathrm{LS}_{j},
\end{aligned}
$$

where the term $S_{k_{m}}$ must be evaluated according to Eq. (26) in which $m$ and $N$ must be replaced by $\left|N_{j}-N_{s}\right|$ and $\max \left(N_{j}, N_{s}\right)$, respectively. 


\subsection{Problem formulation}

For the sake of simplicity, only quasi-homogeneous, fully orthotropic laminates [26, 27, $32,33]$ will be considered in the following. A laminate is said quasi-homogeneous when it is uncoupled $\left[\mathrm{B}^{*}\right]=[\mathrm{O}]$ and when homogenised membrane and bending matrices are equal, i.e. $\left[\mathrm{A}^{*}\right]=\left[\mathrm{D}^{*}\right]$. Quasi-homogeneous laminates can be easily obtained by using general quasi-trivial stacking sequences which represent exact solutions with respect to the quasihomogeneity condition, regardless of the value of the plies orientations. More details on quasi-homogeneous laminates and quasi-trivial stacks can be found in [19, 26, 27, 32, 33]. Under the hypotheses of quasi-homogeneous and fully orthotropic laminates only four design variables are needed to completely characterise the elastic behaviour of the $j$-th subpanel at the macroscopic scale, i.e. $\left(R_{0 K}^{A *}\right)_{j},\left(R_{1}^{A *}\right)_{j},\left(\Phi_{1}^{A *}\right)_{j}, N_{j}$. The design variables of each sub-panel can be grouped into the following vector:

$$
\boldsymbol{\xi}=\left\{\left(R_{0 K}^{A *}\right)_{j},\left(R_{1}^{A *}\right)_{j},\left(\Phi_{1}^{A *}\right)_{j}, N_{j}\right\}, j=1, \ldots, N_{p} .
$$

In addition, in the formulation of the optimisation problem at the first level of the strategy, the feasibility constraints on the polar parameters (which arise from the combination of the layers orientations and positions within the stack) must also be considered. These constraints ensure that the optimum values of the polar parameters resulting from the first step correspond to a feasible laminate that will be designed during the second step of the MS2L strategy, see [30].

Since the laminate of each sub-panel is quasi-homogeneous, such constraints can be written only for matrix $\left[\mathrm{A}^{*}\right]$ :

$$
\left\{\begin{array}{l}
-R_{0} \leq\left(R_{0 K}^{A *}\right)_{j} \leq R_{0} \\
0 \leq\left(R_{1}^{A^{*}}\right)_{j} \leq R_{1} \\
2\left(\frac{\left(R_{1}^{A^{*}}\right)_{j}}{R_{1}}\right)^{2}-1-\frac{\left(R_{0 K}^{A *}\right)_{j}}{R_{0}} \leq 0 \\
\text { with } j=1, \cdots, N_{p} .
\end{array}\right.
$$

In the previous equation, the first and the second constraint can be taken into account as admissible intervals for the relevant optimisation variables, i.e. on $\left(R_{0 K}^{A *}\right)_{j}$ and $\left(R_{1}^{A *}\right)_{j}$, respectively. Hence, the resulting feasibility constraints for the $j$-th sub-panel is:

$$
g_{j}(\boldsymbol{\xi})=2\left(\frac{\left(R_{1}^{A^{*}}\right)_{j}}{R_{1}}\right)^{2}-1-\frac{\left(R_{0 K}^{A *}\right)_{j}}{R_{0}} \leq 0, j=1, \cdots, N_{p} .
$$

The optimisation problem is then formulated as a standard constrained non-linear pro- 
gramming problem (CNLPP) as follows:

$$
\min _{\boldsymbol{\xi}} \frac{M(\boldsymbol{\xi})}{M_{r e f}}
$$

subject to:

$$
\left\{\begin{array}{l}
\text { feasibility constraints of Eq. (30), } \\
\text { blending constraints of Eq. (16), } \\
1-\lambda_{j} \leq 0 \\
\frac{1}{\mathrm{SF}}-\frac{1}{N_{e j}} \max \left(\frac{\sum_{e \in S_{j}} \varepsilon_{x}^{(e)}}{\varepsilon_{x}^{a}}, \frac{\sum_{e \in S_{j}} \varepsilon_{y}^{(e)}}{\varepsilon_{y}^{a}}, \frac{\sum_{e \in S_{j}} \gamma_{x y}^{(e)}}{\gamma_{x y}^{a}}\right) \leq 0 \\
\text { with } j=1, \cdots, N_{p} .
\end{array}\right.
$$

In Eq. (31), $M(\boldsymbol{\xi})$ is the wing-box mass, while $M_{\text {ref }}$ is a reference value (related to the reference configuration taken from [13]). $\lambda_{j}$ is the buckling factor computed, via a global-local approach, for the $j$-th dorsal sub-panel, while $\varepsilon_{x}^{(e)}, \varepsilon_{y}^{(e)}$ and $\gamma_{x y}^{(e)}$ are the laminate strains computed by averaging the strains at the centroids of a sub-set of elements $S_{j}$ (composed of $N_{e j}$ elements) belonging to the $j$-th sub-panel. As shown in Figure 5 , the sub-set $S_{j}$ is located at the centre of each sub-panel. $\varepsilon_{x}^{a}, \varepsilon_{y}^{a}$ and $\gamma_{x y}^{a}$ are the allowable strain values and $\mathrm{SF}$ is a suitable safety factor. These quantities are reported in Table 3.

\begin{tabular}{cccc}
\hline$\varepsilon_{x}^{a}$ & $\varepsilon_{y}^{a}$ & $\gamma_{x y}^{a}$ & $S F$ \\
0.08 & 0.029 & 0.015 & 1.5 \\
\hline
\end{tabular}

Table 3: Laminate allowable strains and associated safety factor.

Table 4 gives an overview of the number of optimisation constraints characterising problem (31) when the number of sub-panels is $N_{p}=3$. In this case the overall number of design variables is 12 .

\begin{tabular}{c|c|c}
\hline Type of constraint & Total number of equations & Comments \\
\hline Feasibility & 3 & 1 eq. for each panel \\
\hline Blending & 8 & $\begin{array}{c}\text { eqs. for each couple of } \\
\text { adjacent panels }\end{array}$ \\
\hline Strain & 3 & 1 eq. for each panel panel \\
\hline Buckling & 3 & $\begin{array}{c}\text { Evaluated through a } \\
\text { global-local approach for each panel }\end{array}$ \\
\hline
\end{tabular}

Table 4: Number and nature of optimisation constraints.

The design space (for the generic sub-panel) of the first-level problem, together with the type of each design variable, is detailed in Table 5. It is noteworthy that, when the laminate is quasi-homogeneous, only blending constraints of Eq. (16) must be integrated into the optimisation problem formulation because they represent the most limiting condition. Indeed, it can be easily proved by comparing Eq. (27) to Eq. (16) that the following 


\begin{tabular}{cc|c|c|c|c}
\hline \multicolumn{2}{c|}{ Variable } & Lower bound & Upper bound & Discretisation & Type \\
\hline$N$ & {$[-]$} & 40 & 150 & 1 & Discrete \\
$R_{0 K}^{A^{*}}$ & {$[\mathrm{MPa}]$} & $-R_{0}$ & $R_{0}$ & - & Continuous \\
$R_{1}^{A^{*}}$ & {$[\mathrm{MPa}]$} & 0 & $R_{1}$ & - & Continuous \\
$\Phi_{1}^{A^{*}}$ & {$[-]$} & $-90^{\circ}$ & $90^{\circ}$ & $1^{\circ}$ & Discrete \\
\hline
\end{tabular}

Table 5: Design space for problem (31).

property holds:

$$
\frac{S_{k m}\left(\max \left(N_{j}, N_{s}\right),\left|N_{j}-N_{s}\right|\right)}{\max \left(N_{j}^{3}, N_{s}^{3}\right)} \geq \frac{\left|N_{j}-N_{s}\right|}{\max \left(N_{j}, N_{s}\right)} .
$$

\subsection{Numerical strategy}

Problem (31) is a highly non-convex CNLPP in terms of both geometrical and mechanical design variables. Its non-convexity is due to the requirements on the buckling load factor and on the maximum allowable strains as well.

To perform the solution search the ERASMUS (EvolutionaRy Algorithm for optimiSation of ModUlar Systems) optimisation tool is coupled with the ANSYS FE commercial software (to calculate the first buckling factor and the strain field of the structure). The GA ERASMUS has already successfully been applied to solve different kinds of real-world engineering problems, see for example [18, 19, 21, 22, 29].

For each individual generated by the ERASMUS tool, at each iteration, four FE analyses of the wing-box model are carried out: a global static analysis and three local buckling analyses (i.e. one for each panel). The outputs are the values of the objective function and the constraint ones. Then the GA elaborates the results provided by the FE analysis to perform the genetic operations. This loop is repeated until the user-defined convergence criterion is satisfied. A scheme of the numerical strategy is illustrated in Figure 4.

The generic individual represents a potential candidate solution of problem (31). Its genotype is characterised by three chromosomes (one for each sub-panel) each one composed of four genes, where each gene is related to a design variable of the panel according to Eq. (28).

Figure 5 further clarifies the FE analysis phase of the numerical strategy of Figure 4 . This phase involves a global linear static analysis followed by three local eigenvalue buckling analyses for each sub-panel via a global/local approach. Both global and local FE models allow for the computation of the objective function and some of the constraint functions, i.e. the values of the laminate strains and the buckling factors. The feasibility and the blending constraints are evaluated before the generation of the FE models since, if not respected, they would generate unfeasible or non-manufacturable solutions.

\section{Finite element model}

The FE model of the wing-box, employed in the first-level problem, is automatically generated by means of an ad-hoc Ansys Parametric Design Language (APDL) script. In fact, through the APDL language it is possible to handle the generation of global and locals FE models by using, as input, the information provided by the ERASMUS code, i.e. the vector of design variables (see Figure 4). Moreover, with the same script, both the 


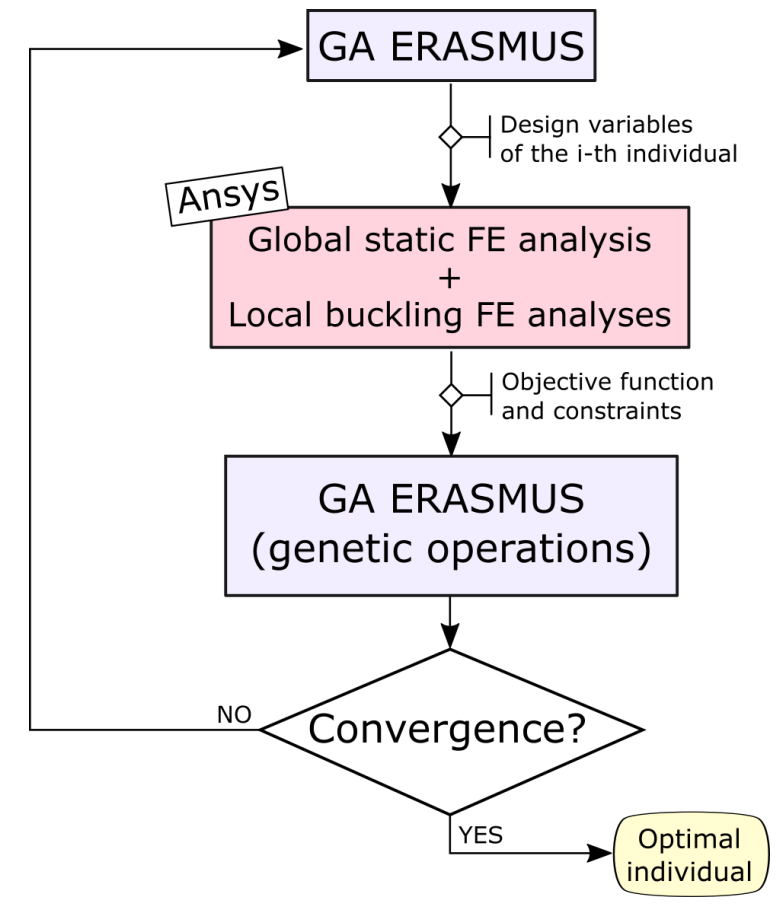

Figure 4: Workflow of the numerical strategy for the first-level problem.

geometry and the mesh of the FE models are automatically generated. The APDL script generates both global and local FE models of the wing-box. In particular the results of the FE analyses are the strain field and the buckling load factor of each sub-panel composing the design region illustrated in Figure 5. The FE analyses are carried out according to the following steps:

1. generation of the global FE model of the wing-box structure, see Figure 6 (a);

2. run of the static analysis on the global FE model, see Figure 6 (b), and evaluation of the strain field in the central region of each sub-panel (as shown in Figure 5);

3. automatic extraction and generation of the local FE model for each sub-panel, see Figure 6 (c);

4. automatic application of the boundary conditions (BCs) (obtained as a result of the static analysis on the global FE model) to each local FE model;

5. evaluation of the buckling factor for each sub-panel.

Four-nodes shell elements (ANSYS SHELL181 elements) have been used to model the global model as well as the local one for a total number of approximately 4500 and 480 elements, respectively, as shown in Figure 6. The selected mesh size $(80 \mathrm{~mm})$ was calibrated through a sensitivity study (not reported here for the sake of synthesis) in order to find a compromise between accuracy and computational costs.

While the global FE model is subject to the same boundary conditions (BCs) of the reference configuration [13] (illustrated in Figure 5), the three local FE models are generated via a sub-modeling strategy which allows sub-panels to be selected from the global model and to be constrained with the generalised displacements obtained as solution of the linear static analysis of the global model. Since the local models solely consist of the upper wing-box laminates, the boundary, where the generalised displacements are imposed, is composed of edges representing the connections with other structural components (spars, ribs and stringers).

The BCs enforced to both global and local FE models are summarized here below. 


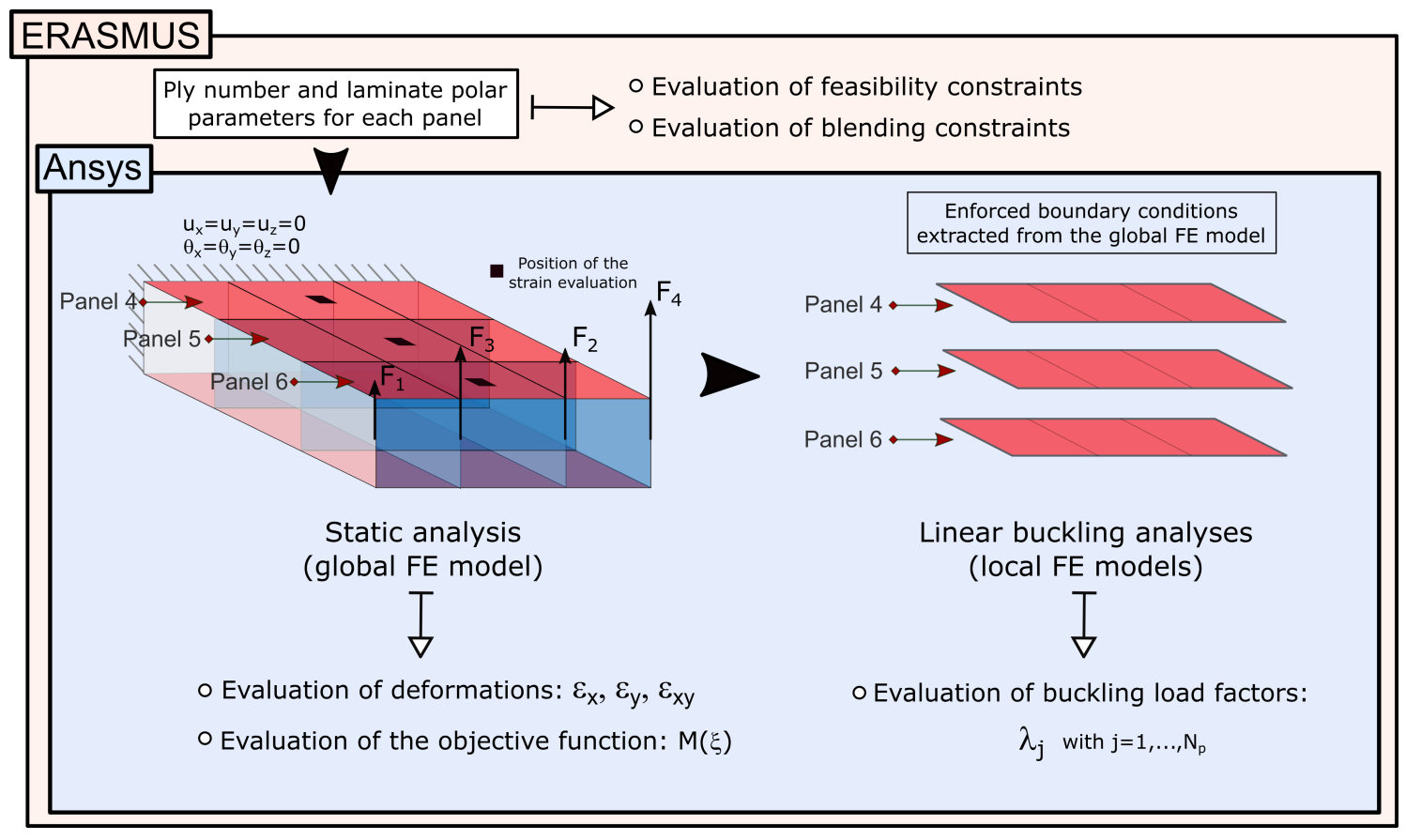

Figure 5: Details of the FE analysis phase.

- Global FE model: $u_{x}=u_{y}=u_{z}=0$ and $\theta_{x}=\theta_{y}=\theta_{z}=0$ for the nodes belonging to the plane $x=0$ and concentrated forces on the opposite end $(x=3543 \mathrm{~mm})$, as shown in Figure 6 (b).

- Local FE models: generalised displacements, solution of the linear static analysis on the global FE model, enforced on the nodes belonging to the intersection lines with spars, ribs and stringers, as shown in Figure 6( c).

\section{Numerical results}

The parameters of the GA ERASMUS, used within the optimisation, are set as reported in Table 6. Optimisation constraints are handled through the Automatic Dynamic Penalisation (ADP) method [34].

\begin{tabular}{c|c}
\hline N. of populations & 2 \\
N. of individuals & 130 \\
N. of generations & 250 \\
Crossover probability & 0.85 \\
Mutation probability & 0.0077 \\
Selection operator & Roulette-wheel \\
Elitism operator & Active \\
\hline
\end{tabular}

Table 6: Genetic parameters of the GA ERASMUS for the first-level problem.

Since problem (31) is non-convex several pseudo-optimal solutions can be retrieved at the end of the optimisation step. The optimal value of the design variables characterising the first three best individuals provided by the ERASMUS code are listed in Table 7 . 


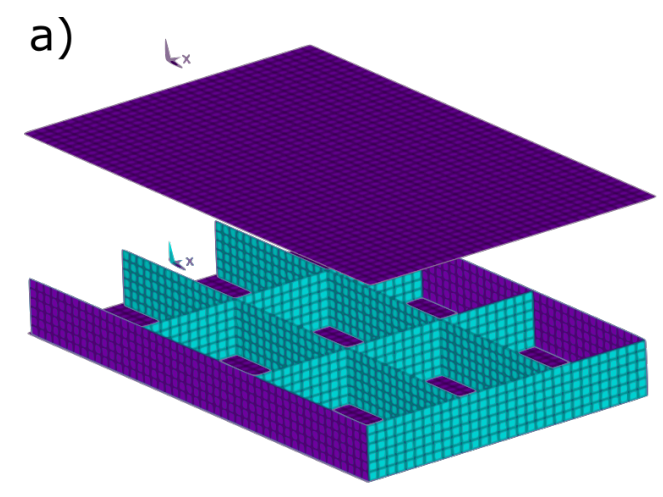

b)

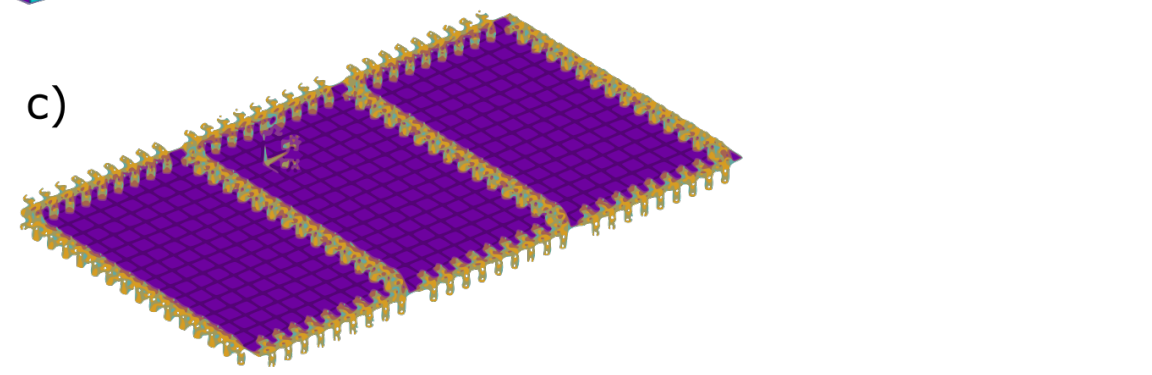

Figure 6: FE models: a) global model, b) BCs applied to the global model and c) BCs applied to one of the local models.

Concerning the best individual, each sub-panel is characterised by a different type of elastic symmetry. In particular, in the solution 1 of Table 7 , panel 4 shows the special $R_{0^{-}}$ orthotropy (since the parameter $R_{0 K}^{A *}$ is one order of magnitude lower than $R_{1}^{A *}$ ) with the main orthotropy axis oriented at $-40^{\circ}$ (the orientation of the orthotropy axis is measured with respect to the global $x$ axis of the wing-box). Panel 5 is characterised by the standard orthotropy with $K^{A^{*}}=1$ (because parameter $R_{0 K}^{A *}$ is negative) and its main orthotropy axis is oriented at $-18^{\circ}$. Finally, Panel 6 is characterised by the standard orthotropy with $K^{A^{*}}=0$ (because parameter $R_{0 K}^{A *}$ is positive) and its main orthotropy axis is oriented at $27^{\circ}$.

It is noteworthy that the main orthotropy axis of each sub-panel is not oriented at $0^{\circ}$ because the wing-box is subject to both torsion and flexural loads. Figure 7 shows the values of the objective function (on the left) and of the buckling factors (on the right) vs. number of generations of the best individuals of each generation.

After 214 generations, the best solution (the three solutions of Table 7 have the same total mass) presents a total mass approximately $12 \%$ lighter $(276.47 \mathrm{~kg})$ than the reference solution (characterised by the stacks of Table 2). As expected, a decrease of the objective function corresponds to a global improvement of the efficiency of the structure. In particular, as shown in Figure 7 (right), the values of the constraints involving the buckling factors of each one of the three panels (see the formulation of problem (31)) are progressively reduced between the first and the last generation: the optimal values of the buckling factors (for the solution 1 of Table 7), of Panel 4, 5 and 6 are 1.015, 1.004 and 1.016, respectively.

\section{Conclusions}

A new formulation of blending constraints has been developed in the context of the MS2L optimisation strategy of composite structures.

In particular, blending constraints have been directly integrated into the formulation of 


\begin{tabular}{ccccc} 
& $N_{\text {ply }}$ & $R_{0 K}^{A^{*}}$ & $R_{1}^{A^{*}}$ & $\Phi_{1}^{A^{*}}$ \\
\hline \multicolumn{5}{c}{ Solution 1 } \\
\hline Panel 4 & 137 & 357.5 & 4392.6 & $-40^{\circ}$ \\
\hline Panel 5 & 110 & -1919.9 & 5338.9 & $-18^{\circ}$ \\
\hline Panel 6 & 80 & 4276.7 & 2641.2 & $27^{\circ}$ \\
\hline \multicolumn{5}{c}{ Solution 2 } \\
\hline Panel 4 & 136 & 198.6 & 4392.6 & $-30^{\circ}$ \\
\hline Panel 5 & 110 & -225.1 & 5310.7 & $-10^{\circ}$ \\
\hline Panel 6 & 81 & 2661.3 & 2641.2 & $35^{\circ}$ \\
\hline \multicolumn{5}{c}{ Solution 3 } \\
\hline Panel 4 & 136 & 410.4 & 4336.1 & $-32^{\circ}$ \\
\hline Panel 5 & 110 & -1919.9 & 4406.7 & $-18^{\circ}$ \\
\hline Panel 6 & 81 & 4594.4 & 2683.6 & $23^{\circ}$ \\
\hline
\end{tabular}

Table 7: The first three optimised configurations of the composite wing-box solutions of problem (31).

the first-level problem which focuses on the laminate macroscopic scale. At this scale the laminate is modelled as an equivalent homogeneous anisotropic plate whose behaviour is described by means of the PPs of the constitutive stiffness matrices in the FSDT framework. Therefore, blending constraints have been formulated as equivalent mechanical constraints in terms of the laminate PPs and number of plies (for both membrane and bending stiffness matrices) to be integrated into the first-level problem formulation regardless of the nature of the stacking sequence.

As a matter of fact, blending constraints have been formulated as equivalent mechanical constraints in terms of the laminate PPs and of the number of plies (for both membrane and bending stiffness matrices) to be integrated into the first-level problem formulation in order to take into account for a general blending scheme. Blending constraints are derived in terms of the maximum variation of the PPs of both membrane and bending matrices. In this background, it is possible to take full advantage of the representation of the elastic symmetries of tensors characterising the polar formalism. In particular, the blending constraints formulation can be really simplified thanks to the properties of the PPS without introducing useless and limiting simplifying hypotheses on the nature of the stack. The effectiveness of the new formulation of the blending constraints, as well as that of the MS2L optimisation strategy, has been proved through a meaningful benchmark taken from the literature: the least-weight design of a composite wing-box structure subject to requirements on blending, on the first buckling load and on the maximum allowable strain. As far as the optimisation calculations are concerned, they are carried out by a special genetic algorithm, i.e. the ERASMUS code, able to integrate both continuous and discretevalued variables during the same calculation and to effectively handle the optimisation constraints by means of the very general ADP method. For the solution of the first-level problem, the GA ERASMUS has been interfaced with the commercial FE code ANSYS that evaluates the objective and the constraint functions by means of a dedicated global/local modelling strategy of the wing-box structure.

The utilisation of an evolutionary strategy allows finding optimised configurations of the 

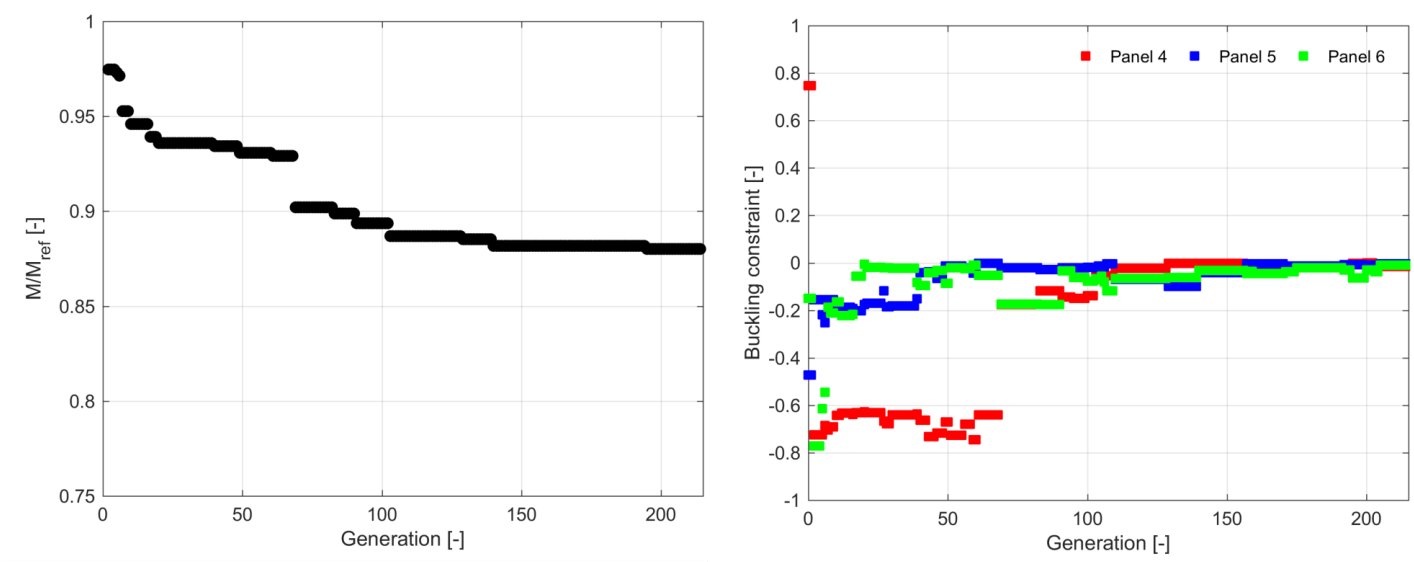

Figure 7: Values of the objective function and of the buckling constraints of the three sub-panels vs. the generation number.

wing-box structure that are more efficient than the reference optimised solution taken from the literature. In fact, the considered example proves that, when standard rules for tailoring laminate stacks are abandoned and if blending constraints are integrated since the first-level problem, a significant weight saving can be obtained: up to $12 \%$ with equivalent mechanical properties in terms of both first buckling load and maximum allowable strain when compared to the reference solution.

As far as the perspectives of this work are concerned, research is ongoing in order to retrieve suitable stacking sequences, as a result of the second-level problem, satisfying the optimised value of both geometric variables and PPs resulting from the first-level problem while ensuring a correct blending between adjacent laminates. The idea is to develop a general strategy without using pre-defined SSTs and by exploiting the interesting properties of a general class of stacking sequences: the so-called quasi-trivial stacks.

\section{Acknowledgements}

This paper presents part of the activities carried out within the research project PARSIFAL (Prandtlplane ARchitecture for the Sustainable Improvement of Future AirpLanes), which has been funded by the European Union under the Horizon 2020 Research and Innovation Program (Grant Agreement n. 723149).

\section{References}

[1] E. Witten, M. Sauer, M. Kühnel, Composites market report 2017, Tech. rep., Carbon Composites (2017).

[2] S. Biggers, S. Srinivasan, Compression buckling response of tailored rectangular composite plates, AIAA Journal 31 (1993) 590-596.

[3] S. Biggers, S. Srinivasan, Postbuckling response of piece-wise uniform tailored composite plates in compression, Journal of Reinforced Plastics and Composites 13 (1994) 803-821.

[4] A. Baranski, S. Biggers, Postbuckling analysis of tailored composite plates with progressive damage, Composite Structures 46 (1999) 245-255. 
[5] L. Papadopoulos, C. Kassapoglou, Shear buckling of rectangular composite plates with two concentric layups, Journal of Reinforced Plastics and Composites 23 (2004) 5-16.

[6] L. Papadopoulos, C. Kassapoglou, Shear buckling of rectangular composite plates composed of concentric layups, Composites Part A: Applied Science and Manufacturing 38 (2007) 1425-1430.

[7] B. Kristinsdottir, Z. Zabinsky, M. Tuttle, S. Neogi, Optimal design of large composite panels with varying loads, Composite Structures 51 (2001) 93-102.

[8] G. Mabson, D. L. Graesser, Cost optimization software for transport aircraft design evaluation (COSTADE), NASA Contractor Report 4738 (August 1996).

[9] O. Seresta, Z. G. and. B. Adams, L. T. Watson, Optimal design of composite wing structures with blended laminates, Composites Part B: Engineering 38 (2007) 469-480. doi:10.1016/j.compositesb.2006.08.005.

[10] B. P. Kristinsdottirt, Z. B. Zabinsky, M. E. Tuttle, S. Neogi, Optimal design of large composite panels with varying loads, Composite Structures 51 (2001) 93-102.

[11] T. Macquart, M. T. Bordogna, P. Lancelot, R. De Breuker, Derivation and application of blending constraints in lamination parameter space for composite optimisation, Composite Structures 135 (2016) 224-235. doi:10.1016/j.compstruct.2015.09. 016.

[12] B. Liu, R. T. Haftka, M. A. Akgun, Two-level composite wing structural optimization using response surfaces, Structural and Multidisciplinary Optimization 20 (2000) 8796.

[13] D. Liu, V. V. Toropov, O. M. Querin, D. C. Barton, Bilevel optimization of blended composite wing panels, Journal of Aircraft 48 (2011) 107-118.

[14] S. Ijsselmuiden, M. M. Abdalla, O. Seresta, Z. Gürdal, Multi-step blended stacking sequence design of panel assemblies with buckling constraints, Composites Part B: Engineering 40 (4) (2009) 329-336.

[15] Z. Jing, X. Fan, Q. Sun, Global shared-layer blending method for stacking sequence optimization design and blending of composite structures, Composites Part B: Engineering 69 (2015) 181-190.

[16] A. Mukherjee, B. Varughese, Design guidelines for ply drop-off in laminated composite structures, Composites Part B: Engineering 32 (2) (2001) 153-164.

[17] D. Cairns, J. Mandell, M. Scott, J. Maccagnano, Design and manufacturing considerations for ply drops in composite structures, Composites Part B: Engineering 30 (5) (1999) 523-534.

[18] M. Montemurro, A. Catapano, On the effective integration of manufacturability constraints within the multi-scale methodology for designing variable angle-tow laminates, Composite Structures 161 (2017) 145-159.

[19] M. Montemurro, A. Pagani, G. Fiordilino, J. Pailhès, E. Carrera, A general multi-scale two-level optimisation strategy for designing composite stiffened panels, Composite Structures 201 (2018) 968-979. 
[20] M. Montemurro, A. Catapano, A general B-Spline surfaces theoretical framework for optimisation of variable angle-tow laminates, Composite Structures 209 (2019) 561578.

[21] M. Montemurro, M. I. Izzi, J. El-Yagoubi, D. Fanteria, Least-weight composite plates with unconventional stacking sequences: Design, analysis and experiments, Journal of Composite Materialsdoi:10.1177/0021998318824783.

[22] M. Montemurro, A. Catapano, Variational Analysis and Aerospace Engineering: Mathematical Challenges for the Aerospace of the Future, 1st Edition, Vol. 116 of Springer optimization and its applications, Springer International Publishing, 2016, Ch. A New Paradigm for the Optimum Design of Variable Angle Tow Laminates, pp. 375-400. doi:10.1007/978-3-319-45680-5_14.

[23] M. Montemurro, A. Catapano, D. Doroszewski, A multi-scale approach for the simultaneous shape and material optimisation of sandwich panels with cellular core, Composites Part B: Engineering 91 (2016) 458-472.

[24] G. Verchery, Les invariants des tenseurs d'ordre 4 du type de l'élasticité, Proc. of colloque Euromech 115, Villard-de-Lans, (France), 1979.

[25] P. Vannucci, Plane anisotropy by the polar method, Meccanica 40 (2005) 437-454.

[26] M. Montemurro, An extension of the polar method to the First-order Shear Deformation Theory of laminates, Composite Structures 127 (2015) 328-339. doi: $10.1016 / j$.compstruct. 2015.03.025.

[27] M. Montemurro, Corrigendum to "An extension of the polar method to the Firstorder Shear Deformation Theory of laminates" [Compos. Struct. 127 (2015) 328-339], Composite Structures 131 (2015) 1143-1144. doi:10.1016/j. compstruct.2015.06. 002.

[28] M. Montemurro, The polar analysis of the Third-order Shear Deformation Theory of laminate, Composite Structures 131 (2015) 775-789. doi:10.1016/j.compstruct. 2015.06 .016 .

[29] M. Montemurro, A contribution to the development of design strategies for the optimisation of lightweight structures, Hdr thesis, Université de Bordeaux (2018).

[30] P. Vannucci, A note on the elastic and geometric bounds for composite laminates, Journal of Elasticity 112 (2013) 199-215. doi:10.1007/s10659-012-9406-1.

[31] S. Tsai, T. Hahn, Introduction to Composite Materials, Technomic, 1980.

[32] P. Vannucci, G. Verchery, A special class of uncoupled and quasi-homogeneous laminates, Composites Science and Technology 61 (10) (2001) 1465-1473.

[33] T. Garulli, A. Catapano, M. Montemurro, J. Jumel, D. Fanteria, Quasi-trivial stacking sequences for the design of thick laminates, Composite Structures 200 (2018) 614-623.

[34] M. Montemurro, A. Vincenti, P. Vannucci, The Automatic Dynamic Penalisation method (ADP) for handling constraints with genetic algorithms, Computer Methods in Applied Mechanics and Engineering 256 (2013) 70-87. 